\title{
Do dispersing non-reproductive female Damaraland mole-rats, Cryptomys damarensis (Rodentia: Bathyergidae) exhibit spontaneous or induced ovulation?
}

\author{
P.C. Snyman, C.R. Jackson and N.C. Bennett
}

\begin{abstract}
The Damaraland mole-rat is a eusocial, subterranean rodent that exhibits a seasonal breeding. Non-reproductive females show physiological suppression of reproduction whilst in the confines of the natal colony. This study set out to investigate whether dispersing female Damaraland mole-rats exhibit induced or spontaneous ovulation. Fifteen non-reproductive females were removed from their natal colonies and housed individually for a period of 6 weeks. During this period urine was collected from all animals every second day. After this initial period the animals were divided into 3 groups. Females were subjected to 1 of 3 trials: a control group housed separately without a male, allowed non-physical contact, or placed in direct physical contact with vasectomized males. Urine was collected for a further 5 weeks, and urinary progesterone profiles established. All three groups showed a significant difference in urinary progesterone concentrations between the two treatment periods indicating initiation of follicular development in all animals following removal from the colony. Histological results further revealed that at least one animal in the control group and five of the six animals in the vasectomized group had corpora lutea present in the ovaries showing that ovulation has taken place. All groups had similar numbers of Graafian follicles, indicating that all females were likely to ovulate in the near future. This finding suggests that females are capable of spontaneous ovulation, but the act of coitus may advance the onset of ovulation in this arid dwelling mole-rat.
\end{abstract}

\section{Article Outline}

1. Materials and methods

1.1. Experimental design

1.2. Surgery

1.3. Sample collection

1.4. Progesterone determination

1.5. Assay validation

1.6. Creatinine determination

1.7. Ovarian histology

1.8. Statistical analysis

2. Results

2.1. Baseline period

2.2. Experimental manipulations 


\subsection{Histology}

3. Discussion

Acknowledgements

References

Ovulation in mammals may be by one of two methods, spontaneous or induced [1]. Females exhibiting induced ovulation show a spontaneous growth and the subsequent development of follicles, but without copulation, they will not ovulate, despite having elevated oestradiol concentrations resulting from the follicular development and subsequent maturation. Spontaneous ovulation on the other hand is characterized by a continuous cycling of reproductive hormones and subsequent ovulation with or without the physical and/or chemical cues of a male [2].

In induced ovulation, cycling of reproductive hormones and subsequent ovulation will only occur in the physical presence of a male (whether the stimulus is tactile, vaginal, olfactory, visual, auditory or perineal) [3]. In truly induced ovulators this physical presence always involves coitus [3]. Solitary subterranean rodents appear to be induced ovulators, i.e. Ctenomys talarum [4] and this seems to be the general rule in nongregarious mammals [5] being an adaptive trait in species relying on brief, chance encounters for mating.

In social, seasonally breeding bathyergids occurring in environments where rainfall is predictable, dispersing females of the highveld mole-rat, Cryptomys hottentotus pretoriae[6] and Natal mole-rat, Cryptomys hottentotus natalensis [7] also exhibit induced ovulation. This is in marked contrast to the eusocial naked mole-rat, Heterocephalus glaberwhich occurs in ecologically constrained environments where rainfall often fails or is sporadic and spontaneous ovulation is the modus operandi [8]. The Damaraland mole-rat also occurs in arid regions of southwestern and South Africa and like the naked mole-rat breeds throughout the year with dispersal events for individuals being few and far between [9].

Non-reproductive female Damaraland mole-rats are physiologically suppressed from reproducing by the reproductive female. In the presence of the reproductive female, the concentrations of luteinising hormone are low and the females remain anovulatory. Removal of the queen from the colony removes this suppression, yet the nonreproductive females will only reproduce with unrelated males usually resulting at dispersal times. Non-reproductive females have few opportunities to disperse and escape the social suppression of reproduction from the breeding animals outside of the natal colony [10]. Damaraland mole-rats show extreme reproductive skew with a single female responsible for procreation and non-breeding female subordinates remaining physiologically suppressed and philopatric to the colony [9]. On dispersing, nonreproductive female Damaraland mole-rats are relieved of suppression and subsequently pair with genetically unrelated dispersing males to form new colonies [9] and [11]. 
Our a priori prediction is that the Damaraland mole-rat should exhibit spontaneous ovulation. Induced ovulation is an adaptive strategy in all mole-rat species studied to date where dispersal is unpredictable or seasonal, as females can be readily stimulated to ovulate as and when they meet a male. Spontaneous ovulation may be better suited in species where physiological suppression is orchestrated on subordinate females by the dominant female. Rainfall events are sporadic and unpredictable in the regions inhabited by the Damaraland mole-rat. Subsequent to these rainfall events there is dispersal of animals from colonies and unrelated males may enter existing colonies and mate with the queen. In such situations multiple paternity of litters results from unrelated male breeding with the queen [12]. If social suppression was not orchestrated on the subordinate females then the opportunity for subordinate breeding in these colonies would be higher as a result of males attempting to mate with subordinates on entering the colony during periods of favourable rainfall.

We simulated dispersal from colonies by removing females from the physical presence of the dominant pair and related non-reproductive animals that form the colony. We subsequently paired some of the females with vasectomized unrelated males to pose a simple but fundamental question: is the eusocial Damaraland mole-rat a spontaneous or induced ovulator?

\section{Materials and methods}

\subsection{Experimental design}

Animals used in the study were captured at Hotazel $\left(27^{\circ} 17^{\prime} \mathrm{S} ; 22^{\circ} 58^{\prime} \mathrm{E}\right)$ in the northern Cape, South Africa during 2000-2001. Complete reproductively functional colonies of between 6 and 15 animals in a colony were trapped with modified Hickman live-traps [13]. All females used in the study had a mass exceeding $60 \mathrm{~g}$ to assure sexual maturity. Non-reproductive females were removed from these wild caught colonies and were housed individually in plastic containers $(1 \times 0.5 \times 0.5 \mathrm{~m})$ in temperature-controlled rooms at the department of Zoology and Entomology, University of Pretoria, South Africa. Temperature in the rooms was maintained between 26 and $28{ }^{\circ} \mathrm{C}$, with a relative humidity of $50 \%$ and a lighting regime of $14 \mathrm{~L}: 10 \mathrm{D}$. Wood shaving served as nesting material. Mole-rats were fed sweet potatoes and chopped vegetables daily, no water was provided since they obtain all of their water requirements from the food resource to maintain a positive water balance.

Experimental manipulations and sampling took place during 2001. The experimental animals were taken from the functionally complete wild captured colonies that comprised a reproductive pair and a number of non-reproductive male and female mole-rats. Nonreproductive females were discerned by their non-perforate vagina and used for the experiment. The Damaraland mole-rat breeds throughout the year and consequently this study was not confined to a particular time of the year. Experimental animals were divided into 2 groups, which had corresponding treatments. Sample collection for the first group ( 6 females) ran from February-May, while sample collection in the second group (6 females) ran from July-October. The non-reproductive females were placed in 
isolation of possible chemical and behavioural stimulation of males for a period of 6 weeks (acclimatization period modeling dispersion). Separated females were housed in plastic containers with dimensions of $50 \times 30 \times 30 \mathrm{~cm}$. This 6-week period was chosen for all experiments, since the oestrous cycle of the naked mole-rat has been estimated to be around one month ( \pm 34 days) [8] and we assumed a similar length would be applicable to the species under study. Samples were taken every second day for the full duration of the initial 6 weeks, these samples thus constituted the baseline samples to enable the animals to be relieved of any suppressive effects of being housed in a colony where reproduction is suppressed. Following the initial 6-week period the animals were divided into 3 groups. The first group ( 3 animals) was left in isolation as control animals and marked $\mathrm{C} 1-\mathrm{C} 3$. The second group (6 animals) was placed in non-physical contact with large, gonadally intact males. Holding cages were provided with a wire-mesh $(2 \mathrm{~mm}$ grid) separation preventing any physical contact, but allowing chemical communication. These animals were marked (S1-S6). The females of the final group (6 animals) were placed in a holding chamber with vasectomized males and allowed to behaviourally interact. In all cases mating took place within 15 min of first contact. The final group was marked V1-V6. In all groups urine was collected every second calendar day.

\subsection{Surgery}

Six males were vasectomized three months prior to being placed in direct physical contact with the six females by a qualified veterinarian. All vasectomies were completed three months prior to pairing up with females to ensure clearance of sperm from the vas deferens as well as a full physical recovery after surgery. Males were anaesthetized using iso-fluorane gas induction and maintenance with a mask. The vas deferens and epididymis were removed from each testis. Vasectomy was only performed in males to be placed into physical contact with a female to prevent fertilization from taking place, such that any resulting corpora lutea would be of ovulation and not pregnancy.

\subsection{Sample collection}

Females were placed in urine collection chambers every second day between $09 \mathrm{~h} 00$ and $14 \mathrm{~h} 00$ during which urine samples were collected. The chambers were cylindrical and had a smooth mesh floor to allow urine to fall through to a collecting dish whilst not allowing faecal contamination. Animals were checked regularly throughout the day, urine samples were collected by pipette and stored in plastic vials marked with the animal number and date. Plastic vials were immediately removed and stored at $-40{ }^{\circ} \mathrm{C}$. All urine collected from a single female was combined for the sampling day. Females were placed back into containers as soon as an adequate sample had been collected. Each female was fed the same amount of food at the same time each day. Urine volume collected each sample day varied (due to variable indirect fluid intake), but usually in the excess of the required amount for analyses $(240 \mu \mathrm{l})$. In the event of no urine being voided by an animal on the sampling day, it was treated as a missed data point, this happened infrequently and had little effect on the overall profiles. 


\subsection{Progesterone determination}

Progesterone is a valuable indicator hormone since it only rises with increasing follicular development and ovulation [14] and plays an important role in the preparation for and maintenance of pregnancy [15]. The cyclical luteal pattern of progesterone secretion constitutes the rationale behind the well-established use of progesterone measurements as a simple and reliable method for ovulation detection [16].

\subsection{Assay validation}

All urine samples were analyzed for progesterone. Progesterone assays were performed as described by Bennett et al. [17] using a coat-a-count progesterone kit (Diagnostic Products Corporation, Los Angeles). The antiserum is highly specific for progesterone with cross-reactivity to all naturally occurring steroids $<0.5 \%$, with the exception of $17 \alpha$

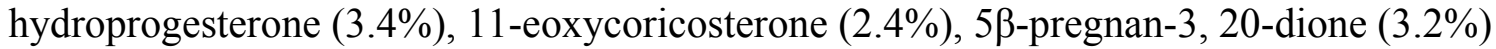
and $5 \alpha$-pregnan-3, 20-dione $(9.0 \%)$. Standard concentrations ranged from 0.3 to 127.2 $\mathrm{nmol} / \mathrm{l}$.

The assay was validated for $C$. damarensis plasma by testing the slope of the curve produced using serial dilutions of un-extracted mole-rat plasma obtained from a pregnant female (over the range $1: 1$ to $1: 64$ ) against that of the standard curve. Following logit$\log$ transformation of the data [18], the slopes of the lines were compared using the Statistica computer package [19] and found not to differ significantly (ANCOVA, $F_{1-}$ $\left.{ }_{6}=4.9, P>0.05\right)$ [20]. The minimum detection limit of the assay was $0.36 \mathrm{nmol} / 1$. Intraand inter-assay coefficients of variation were $4.4 \%(n=20)$ and $6.6 \%(n=4)$, respectively.

\subsection{Creatinine determination}

Prior to hormone assay all urine samples are subjected to a determination of creatinine [21]. Creatinine is a breakdown product of tissue proteins, usually formed in the muscles of mammals [22] and as such is excreted at a constant rate. Expressing urine hormone concentrations per milligram CR takes account of the differing dilutions of urine, which may be produced by animals according to their fluid intake. Progesterone concentrations are therefore expressed as ng progesterone/mg creatinine.

Creatinine concentration for each of the samples was calculated using a modified Jaffe reaction [23]. The process involves adding $10 \mu \mathrm{l}$ of standard or sample to the wells of a microplate, in duplicate, and leaving two wells empty as a duplicate control blank. A further $300 \mu \mathrm{l}$ of picrate reagent was added to all wells, including the blanks.

Fresh alkaline picrate was mixed and comprised a saturated picric acid solution, alkaline triton and distilled, deionized $\mathrm{H}_{2} \mathrm{O}(1: 1: 10)$. The alkaline triton is composed of $4.2 \mathrm{ml}$ triton $\mathrm{x}-100,12.5 \mathrm{ml} 1 \mathrm{~N} \mathrm{NaOH}$ and $66.0 \mathrm{ml}$ distilled, deionised $\mathrm{H}_{2} \mathrm{O}$. The microplate is then placed in the dark for a period of $1.5 \mathrm{~h}$, at room temperature, to allow colour 
development to occur. A standard curve $\left(R^{2}>0.99\right)$ was used to determine all sample values.

\subsection{Ovarian histology}

Animals were weighed and deeply anaesthetized with halothane. Both ovaries were dissected out and then placed in Bouin's fixative for $24 \mathrm{~h}$ after which they were rinsed and stored in $70 \%$ ethanol. The reproductive tracts were sequentially dehydrated and embedded in paraffin wax using standard histological techniques. The ovaries were serially sectioned at $4 \mu \mathrm{m}$. Both ovaries of each female were entirely mounted on glass slides. The sections were stained with Ehrlich's haematoxylin, counter-stained with eosin [24] and examined under a standard light microscope.

The analysis was primarily concerned with the presence or absence of follicular development and corpora lutea production in the ovaries of the females. Bloom and Fawcett [25] and Bennett et al. [17] were used as a reference to identify and categorize follicles at various stages of maturity.

\subsection{Statistical analysis}

Transformation of data provided mean progesterone values (ng progesterone per $\mathrm{mg}$ creatinine) as well as SD for both before and after animals were paired for each of the 3 experimental groups. These values were subjected to non-parametric analyses of variance (ANOVA) test using Statistica software package [19].

\section{Results}

The Damaraland mole-rat is without doubt a spontaneous ovulator. Both the progesterone hormone profiles (Fig. 1, Fig. 2 and Fig. 3) and the histological findings support the notion that ovulation can occur spontaneously in non-reproductive females, although recrudescence of ovulation in these previously socially suppressed females is enhanced in the presence of a non-related breeding male (Fig. 3 and Table 1).

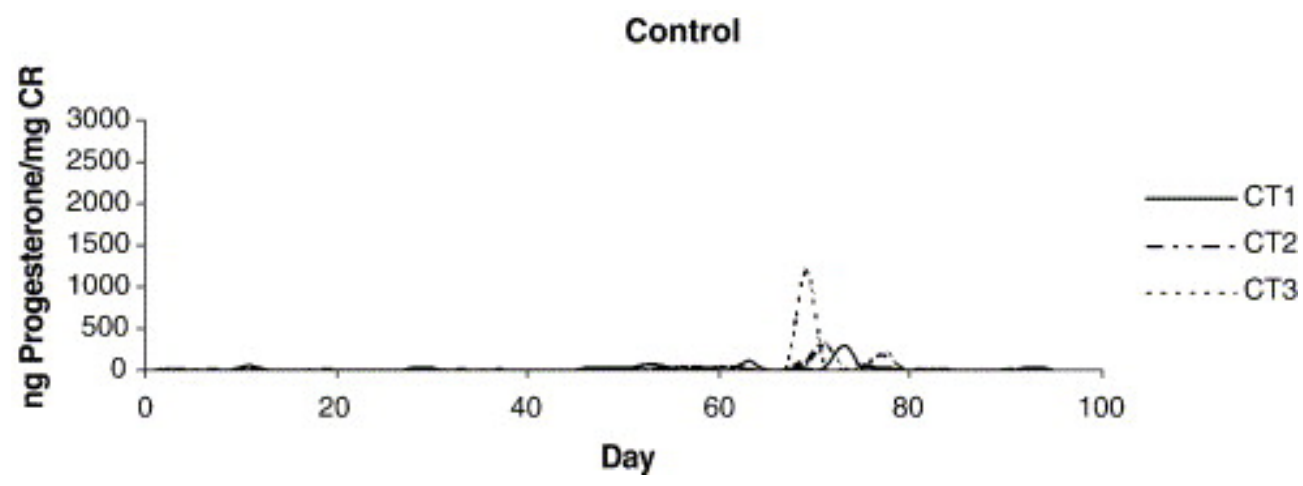

Fig. 1. Urinary progesterone profile (ng/mg creatinine) for each of three female Damaraland mole-rats housed singly. 
Non-physical Contact

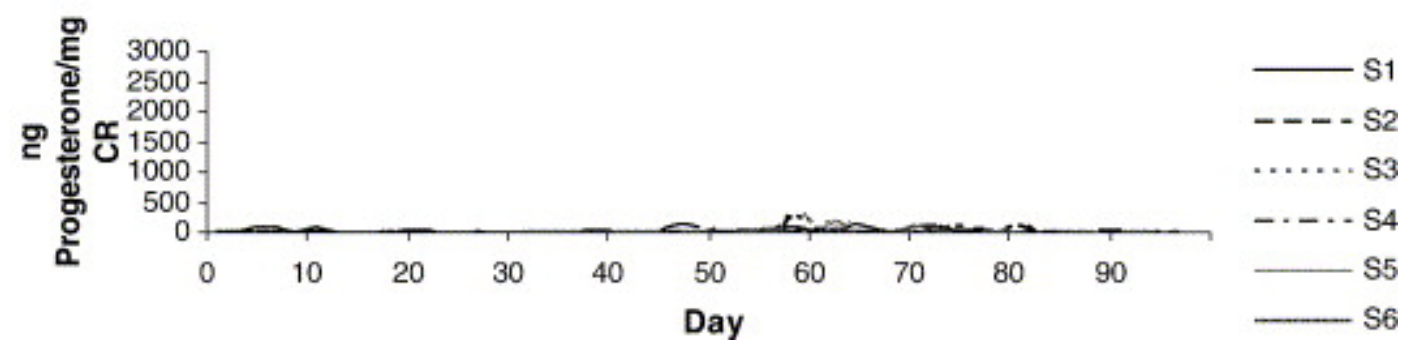

Fig. 2. Urinary progesterone profile ( $\mathrm{ng} / \mathrm{mg}$ creatinine) for each of six female Damaraland mole-rats housed in non-physical contact with a gonadally intact male.

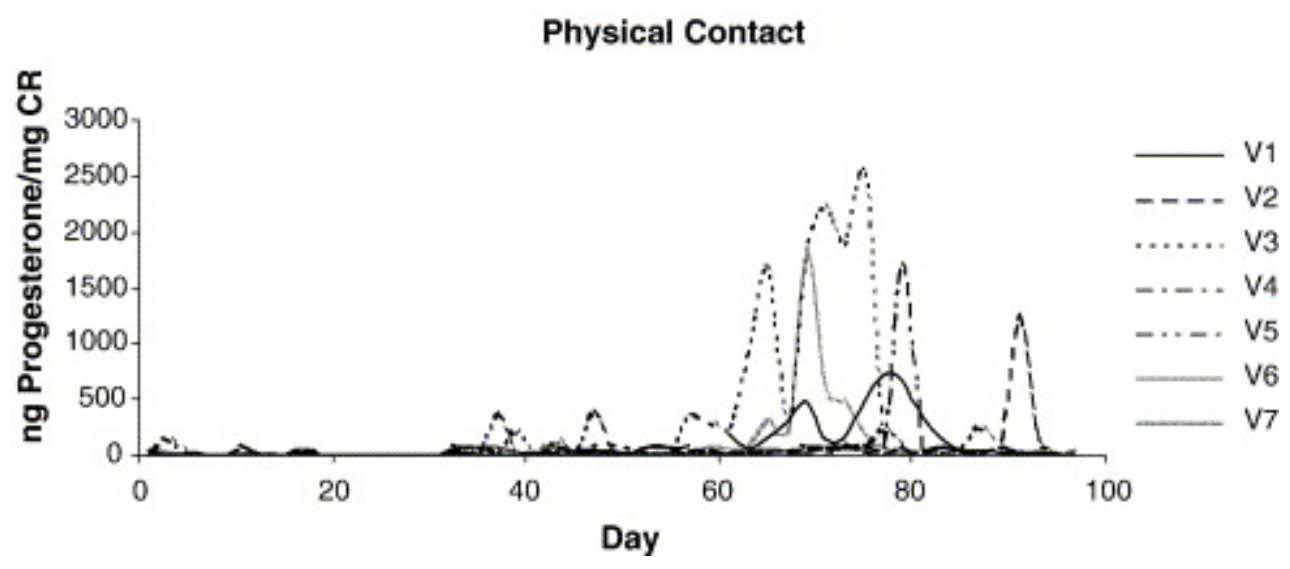

Fig. 3. Urinary progesterone profile ( $\mathrm{ng} / \mathrm{mg}$ creatinine) for each of six female Damaraland mole-rats housed in physical contact with a vasectomized male. 
Table 1.

Results of qualitative histological analysis showing the presence or absence of various stages of follicular development

\begin{tabular}{|c|c|c|c|c|c|}
\hline Treatment & $\begin{array}{l}\text { Anim } \\
\text { al }\end{array}$ & $\begin{array}{l}\text { Primor } \\
\text { dial }\end{array}$ & $\begin{array}{l}\text { Prima } \\
\text { ry }\end{array}$ & $\begin{array}{l}\text { Graafi } \\
\text { an }\end{array}$ & $\begin{array}{l}\text { Corpo } \\
\text { ra } \\
\text { lutea }\end{array}$ \\
\hline \multirow[t]{3}{*}{ Control group } & $\mathrm{C} 1$ & + & + & + & \\
\hline & $\mathrm{C} 2$ & + & + & + & \\
\hline & $\mathrm{C} 3$ & + & + & + & + \\
\hline \multirow[t]{6}{*}{ Separated group (non-physical contact) } & $\mathrm{S} 1$ & + & + & & \\
\hline & $\mathrm{S} 2$ & + & + & + & \\
\hline & $\mathrm{S} 3$ & + & + & & \\
\hline & $\mathrm{S} 4$ & + & + & + & \\
\hline & S5 & + & + & + & \\
\hline & S6 & + & + & + & \\
\hline \multirow{6}{*}{$\begin{array}{l}\text { Vasectomized group (physical contact } \\
\text { including coitus) }\end{array}$} & V1 & + & + & & + \\
\hline & $\mathrm{V} 2$ & + & + & + & + \\
\hline & V3 & + & + & + & + \\
\hline & V4 & + & + & + & + \\
\hline & V5 & + & + & & \\
\hline & V6 & + & + & & + \\
\hline
\end{tabular}

\subsection{Baseline period}

The mean urinary progesterone concentrations in non-reproductive females removed from the presence of the colony were significantly different during the baseline period (Kruskal Wallis $H_{(2,15)}=6.9 ; P=0.032$ ) (Fig. 4). Progesterone concentrations of the control group were lower than the other two groups, but this could be the result of a sample size effect. 


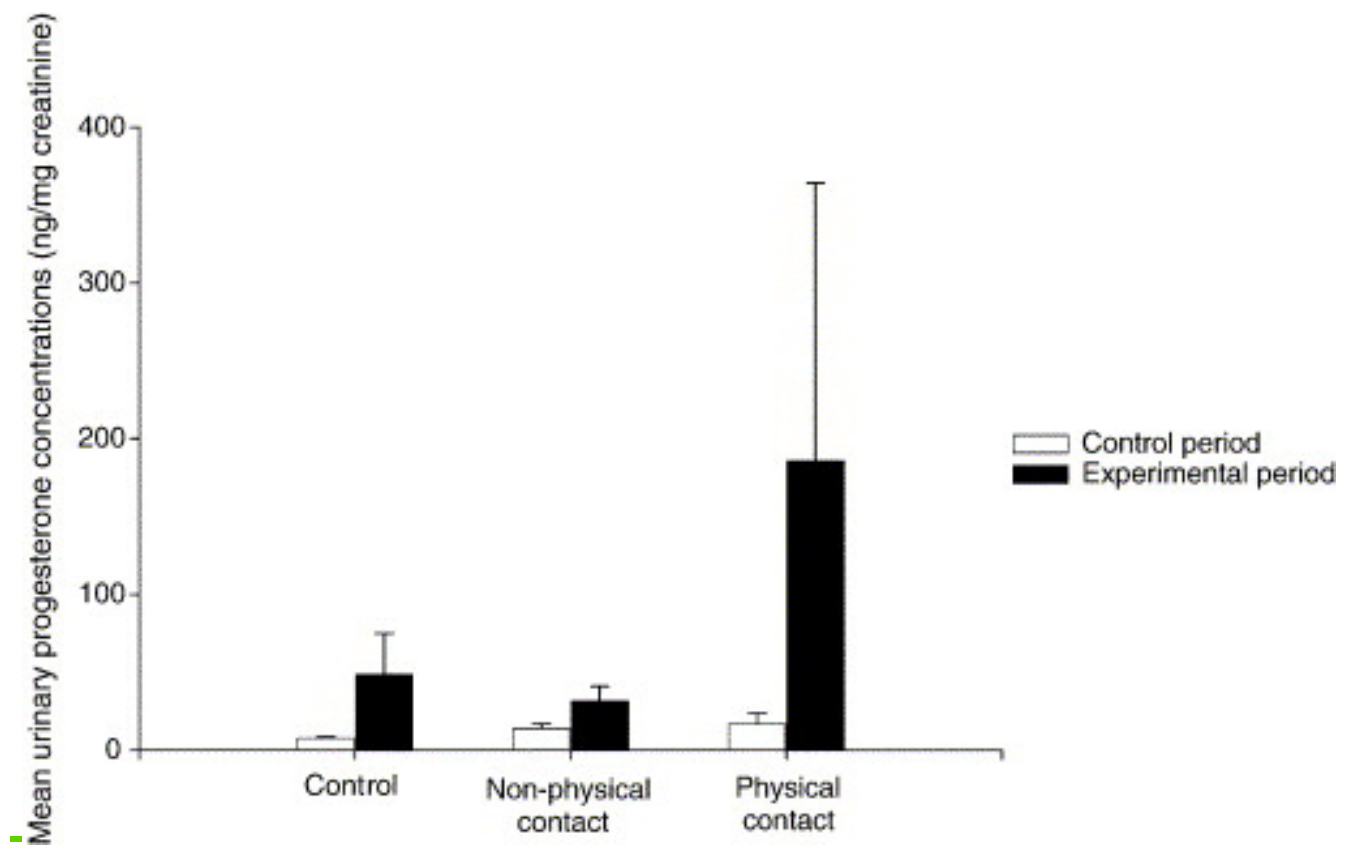

Fig. 4. Mean urinary progesterone concentrations (ng/mg creatinine) before and after experimental manipulation for females housed singly, in non-physical contact with a male and physical contact with a vasectomized male.

Pairwise comparisons revealed that during the initial 6-week period of separation from the colony, the control group had significantly lower progesterone concentrations than those females prior to non-physical contact $(U=0, P=0.02)$ or those in physical contact $(U=0, P=0.02)$ Mann Whitney $U$-test (Fig. 4).

\subsection{Experimental manipulations}

There is a significant difference in progesterone concentrations in both the non-physical Wilcoxon matched pairs test $(Z=2.21 ; P=0.028)$ and physical $(Z=2.20 ; P=0.02)$ groups when comparison is made between progesterone concentrations recorded for the baseline period with those of experimental period (Fig. 4). Furthermore, there is strong support to suggest that elevated levels of progesterone concentrations only really start around day $45-50$ of the experiment.

If one compares the mean progesterone concentration and variance between the three groups during the experimental period it is found that there is no significant difference in progesterone concentration (Kruskal Wallis test $H_{(2,15)}=5.48, P=0.65$ ) (Fig. 4).

\subsection{Histology}

All individuals had primordial and primary follicles present. Graafian follicles were found in all 3 groups. Interestingly, some females in groups S and V had ovaries that 
lacked Graafian follicle production (Table 1). Corpora lutea were found in several of the females. Female $\mathrm{C} 3$ in the control colony had corpora lutea indicating that this animal had ovulated. None of the animals in Group S possessed corpora lutea. Five of the 6 females in Group V had corpora lutea in their ovaries (Table 1).

\section{Discussion}

Numerous families of rodents have adopted a totally subterranean existence and as such there has been considerable parallel convergent evolution among subterranean mammals even though they exhibit divergent evolutionary histories [26]. African mole-rats have proven to be a particularly interesting group to study because of the wide range of social organization and reproductive strategies found within the family [27] and [28].

Induced ovulation appears to be a general rule in non-gregarious mammals [5], being an adaptive trait in solitary species that rely on brief chance encounters for mating and subsequent procreation. Furthermore, several studies have shown this to be true in subterranean rodents as well. Among the Ctenomyidae, C. talarum is known to be an induced ovulator [4] whereas Shanas et al. [29] found Spalax ehrenbergi to be an induced ovulator. The solitary Cape mole-rat, Georychus capensis, has recently been shown to be an induced ovulator [30]. The two sexes from this species meet very briefly for procreation. It has been speculated upon that the common mole-rat, Cryptomys hottentotus hottentotus [31] may be an induced ovulator, while studies on the highveld mole-rat, $C$. hottentotus pretoriae and the Natal mole-rat, $C$. hottentotus natalensis, have revealed that females tend to undergo induced ovulation [6] and [7]. In marked contrast, the naked mole-rat is a spontaneous ovulator [8]. The finding of the present study suggests that non-reproductive female Damaraland mole-rats removed from the social influence of the dominant reproductive female will undergo spontaneous ovulation.

In contrast to two studies on highveld and Natal mole-rats where it was shown that there was a significant difference in progesterone concentration between the control group and the group within non-physical contact with a male [6] and [7] no such pattern was observed in the Damaraland mole-rat. Furthermore a brief glance at the urinary progesterone profiles of all three groups reveals that a time of 45-50 days separation is required prior to spontaneous ovulation taking place. The mean progesterone concentrations for females paired with vasectomized males intimated that coitus enhances the onset of spontaneous ovulation.

Histological examination revealed that follicular development had taken place in all animals, with all groups showing a presence of primordial, primary and Graafian follicles. Non-reproductive females within intact breeding groups have primordial, primary and secondary follicles, with high numbers of atretic follicles, but generally lack more advanced follicular development. A delay in ovulation in removed nonreproductive females may result from the fact that in nature following good rainfall and subsequent dispersal and pairing with an unrelated male an elevation of LH in the pituitary as a result of priming from the GnRH neurons was stimulated by the pairing of these females with males prior to reproduction [32]. In the vasectomized group, 5 out of 
the 6 females showed signs of ovulation. All females paired with vasectomized males participated in mating bouts within $15 \mathrm{~min}$ of initial contact. The act of mating and physical contact with unrelated males therefore seems to greatly enhance the speed of follicular development, which can be seen in both the hormonal profiles and histological findings.

Two non-reproductive female Damaraland mole-rats removed from the confines of their natal colony and housed separately in rooms for six months and subsequently sacrificed possessed corpora lutea in their ovaries, further supporting the notion that ovulation is spontaneous (N.C. Bennett \& A.J. Molteno, unpubl. data).

The results show an interesting trend within the group of social bathyergids. Both of the eusocial species, the naked and Damaraland mole-rat, show spontaneous ovulation, while all of the strictly solitary and social seasonal breeders studied to date undergo induced ovulation. The eusocial Damaraland and naked mole-rat are divergent within the family indicating gains and/or losses of sociality, with associated changes in the control of ovulation [27]. Interestingly, the males from the two eusocial social species lack elaborate ornamentation on their penises. All currently studied solitary and social molerats in the family are induced ovulators and the males of the various species possess epidermal spines or ornamented penises [33]. This study strongly emphasizes the propensity of sexual selective forces shaping ovulation in response to the degree of sociality and morphological ornamentation of the penis. This fits in well with a recent phylogeography of the family that suggests the ancestral bathyergid was from East Africa and that subsequent migration and speciation arose as a result of a southerly migration of bathyergids into southern and central Africa [34]. Many of these southern African species are seasonally breeding, solitary or loosely social and all without exception studied so far exhibit induced ovulation. It is feasible that the ancestral bathyergids were spontaneous ovulators and with their migration into seasonal habitats and the loss of sociality they adopted a pattern of induced ovulation to facilitate rapid reproduction and increase reproductive success.

It is of particular interest that within the genus Cryptomys there are representative species that exhibit both induced and spontaneous ovulatory patterns. This study has provided unequivocal evidence that the Damaraland mole-rat is a spontaneous ovulator. The incidence of spontaneous ovulation in the Damaraland mole-rat may be explained in terms of this species possessing philopatric non-reproductive females that are reproductively suppressed whilst in the confines of the natal colony [9] and [10]. In both Damaraland and naked mole-rats where the queen physiologically suppresses reproduction in non-reproductive females, it appears that the mechanisms for induced ovulation are absent, and control of egg release is spontaneous. This difference may have arisen as a result of the neuroendocrine changes associated with the socially induced suppression of ovulation characteristic of these species [35]. 


\section{References}

[1] S.R. Milligan, Pheromones and rodent reproductive physiology, Symp Zool Soc Lond 45 (1980), pp. 251-275.

[2] E.N.J.D. Knobil, Physiology of reproduction, Raven Press, New York (1988).

[3] V.D. Ramirez and W.K. Soufi, The neuroendocrine control of the rabbit ovarian cycle. In: E. Knobil and J.D. Neill, Editors, The physiology of reproduction. Vol. 2 (Second edition) (1994), pp. 585-612 Chapter 45.

[4] B.J. Weir, Reproductive characteristics of hystricomorph rodents, Symp Zool Soc Lond 34 (1974), pp. 265-301.

[5] M.X. Zarrow and J.H. Clark, Ovulation following vaginal stimulation in a spontaneous ovulator and its implications, J Endocrinol 40 (1968), pp. 343-352.

[6] G.P. Malherbe, N.C. Bennett and A.S. Schoeman, Is the highveld mole-rat, Cryptomys hottentotus pretoriaea induced or spontaneous ovulator?, J Zool Lond $\mathbf{2 6 3}$ (2004), pp. 159-165.

[7] C.R. Jackson and N.C. Bennett, Is the Natal mole-rat (Cryptomys hottentotus natalensis) a spontaneous or induced ovulator?, J Mammal 86 (2005), pp. 1-6.

[8] C.G. Faulkes, D.H. Abbott and J.U.M. Jarvis, Social suppression of ovarion cyclicity in captive and wild colonies of naked mole-rats, Heterocephalus glaber, J Reprod Fertil 88 (1990), pp. 559-568.

[9] J.U.M. Jarvis and N.C. Bennett, Eusociality has evolved independently in two genera of bathyergid mole-rats-but occurs in no other subterranean mammal, Behav Ecol Sociobiol 33 (1993), pp. 353-360.

[10] C.G. Faulkes and N.C. Bennett, Family values: group dynamics and social control of reproduction in African mole-rats, Trends Ecol Evol 16 (2001) (4), pp. 184-190.

[11] T.M. Burland, N.C. Bennett, J.U.M. Jarvis and C.G. Faulkes, Eusociality in African mole-rats: new insights from patterns of genetic relatedness in the Damaraland mole-rat, Proc R Soc Lond B 269 (2002), pp. 1025-1030.

[12] T.M. Burland, N.C. Bennett, J.U.M. Jarvis and C.G. Faulkes, Colony structure and parentage in wild colonies of cooperatively breeding Damaraland mole-rats suggest incest avoidance alone may not maintain reproductive skew, Mol Ecol 13 (2004), pp. 2371-2379.

[13] G.C. Hickman, A live-trap and trapping technique for fossorial mammals, S Afr $J$ Zool 212 (1979), pp. 9-12. 
[14] L.L. Espey and H. Lipner, Ovulation. In: E. Knobil and J.D. Neill, Editors, The physiology of reproduction, Volume 1 (2nd edition), Raven Press, New York (1994), pp. 725-780.

[15] M.B. Aufrere and H. Benson, Progesterone: an overview and recent advances, $J$ Pharm Sci 65 (1976), pp. 783-800.

[16] J. Bauman, Basal body temperature: unreliable method of ovulation detection, Fertil Steril 36 (1981), pp. 729-733.

[17] N.C. Bennett, J.U.M. Jarvis, R.P. Millar, H. Sasano and K.V. Ntshinga, Reproductive suppression in eusocial Cryptomys damarensis colonies: socially-induced infertility in females, J Zool Lond 234 (1994), pp. 617-630.

[18] T. Chard, An introduction to radioimmunoassay and related techniques, Elsevier, Amsterdam (1987) 3rd rev.

[19] STATSOFT StatSoft inc., 6th ed. Tulsa, USA. 2002.

[20] A.J. Molteno and N.C. Bennett, Anovulation in non-reproductive female Damaraland mole-rats (Cryptomys damarensis), J Reprod Fertil 119 (2000), pp. 35-41.

[21] R.C. Bonney, D.J. Wood and D.G. Kleiman, Endocrine correlates of behavioural oestrus in the female giant panda (Ailuropoda melanoleuca) and associated hormonal changes in the male, J Reprod Fertil 64 (1982), pp. 209-215.

[22] K. Schmidt-Nielsen, Animal physiology: adaptation and environment (5th ed.), Cambridge University Press, Cambridge (1997).

[23] O. Folin, Determination of creatinine and creatine in the urine, J Biol Chem 17 (1914), pp. 469-473.

[24] R.A.B. Drury and E.A. Wallington, Carleton's histological technique, Oxford University Press, London (1967).

[25] W. Bloom and D.W. Fawcett, A textbook of history, W.B. Saunders Co., Philadelphia (1962).

[26] E. Nevo, Adaptive convergence and divergence of subterranean mammals, Ann Rev Ecolog Syst 10 (1979), pp. 269-308.

[27] N.C. Bennett and C.G. Faulkes, African mole-rats: ecology and eusociality, Cambridge University Press, Cambridge (2000). 
[28] N.C. Bennett, C.G. Faulkes and A.J. Molteno In: G.N. Cameron, E.A. Lacey and J. Patton, Editors, Reproduction in subterranean rodents in life underground (2000), pp. $145-177$.

[29] U. Shanas, G. Heth, E. Nevo, R. Shalgi and J. Terkel, Reproductive behaviour in the female blind mole (Spalax ehrenbergi), J Zool Lond 237 (1995), pp. 195-210.29.

[30] J. d T. Van Sandwyk and N.C. Bennett, Do solitary, seismic signaling Cape molerats, Georychus capensis demonstrate spontaneous or induced ovulation?, J Zool Lond 267 (2005), pp. 75-80.

[31] A.C. Spinks, N.C. Bennett and J.U.M. Jarvis, Regulation of reproduction in female common mole-rats, Cryptomys hottentotus hottentotus; the effects of breeding season and reproductive status, J Zool Lond 248 (1999), pp. 161-168.

[32] A.J. Molteno and N.C. Bennett, Relaxation of socially-induced reproductive inhibition in colonies of the eusocial Damaraland mole-rat: the effect of aridity as an ecological constraint promoting philopatry, J Zool, Lond 256 (2002), pp. 445-448.

[33] Parag, A., Bennett, N.C., Faulkes, C.G. and Bateman, P.W. (submitted for publication). Penile morphology of African mole-rats (Bathyergidae): structural modification in relation to mode of ovulation and degree of sociality. J. Zool. Lond.

[34] C.G. Faulkes, E. Verheyen, W. Verheyen, J.U.M. Jarvis and Bennett, Phylogeography and speciation in African mole-rats (Family: Bathyergidae), Mol Ecol 13 (2004), pp. 613-629.

[35] N.C. Bennett, C.G. Faulkes and A.J. Molteno, Reproductive suppression in subordinate, non-breeding female Damaraland mole-rats: two components to a lifetime of socially-induced infertility, Proc Roy Soc Series B Lond 263 (1996), pp. 1599-1603. 